University of Nebraska - Lincoln

DigitalCommons@University of Nebraska - Lincoln

10-23-2007

\title{
FePt nanodot arrays with perpendicular easy axis, large coercivity, and extremely high density
}

\author{
Chaehyun Kim \\ University at Buffalo, the State University of New York, Buffalo, New York \\ Thomas Loedding \\ University at Buffalo, the State University of New York, Buffalo, New York \\ Seongjin Jang \\ University at Buffalo, the State University of New York, Buffalo, New York \\ Hao Zeng \\ University at Buffalo, the State University of New York, Buffalo, New York \\ Zhen Li \\ University of Nebraska - Lincoln \\ See next page for additional authors
}

Follow this and additional works at: https://digitalcommons.unl.edu/physicssellmyer

Part of the Physics Commons

Kim, Chaehyun; Loedding, Thomas; Jang, Seongjin; Zeng, Hao; Li, Zhen; Sui, Yucheng; and Sellmyer, David J., "FePt nanodot arrays with perpendicular easy axis, large coercivity, and extremely high density" (2007). David Sellmyer Publications. 207.

https://digitalcommons.unl.edu/physicssellmyer/207

This Article is brought to you for free and open access by the Research Papers in Physics and Astronomy at DigitalCommons@University of Nebraska - Lincoln. It has been accepted for inclusion in David Sellmyer Publications by an authorized administrator of DigitalCommons@University of Nebraska - Lincoln. 


\section{Authors}

Chaehyun Kim, Thomas Loedding, Seongjin Jang, Hao Zeng, Zhen Li, Yucheng Sui, and David J. Sellmyer 


\title{
FePt nanodot arrays with perpendicular easy axis, large coercivity, and extremely high density
}

\author{
Chaehyun Kim, Thomas Loedding, Seongjin Jang, and Hao Zeng a) \\ Department of Physics, University at Buffalo, the State University of New York, Buffalo, \\ New York 14260, USA \\ Zhen Li, Yucheng Sui, and David J. Sellmyer \\ Department of Physics and Nebraska Center for Materials and Nanoscience, University of Nebraska, \\ Lincoln, Nebraska 68588, USA
}

(Received 14 September 2007; accepted 3 October 2007; published online 23 October 2007)

\begin{abstract}
Ordered FePt nanodot arrays with extremely high density have been developed by physical vapor deposition using porous alumina templates as evaporation masks. Nanodot diameter of $18 \mathrm{~nm}$ and periodicity of $25 \mathrm{~nm}$ have been achieved, resulting in an areal density exceeding 1 $\times 10^{12}$ dots/in. ${ }^{2}$. Rapid thermal annealing converts the disordered fcc to $L 1_{0}$ phase, resulting in (001)-oriented FePt nanodot arrays with perpendicular anisotropy and large coercivity, without the need of epitaxy. High anisotropy and coercivity, perpendicular easy axis orientation and extremely high density are desirable features for future magnetic data storage media applications. (C) 2007 American Institute of Physics. [DOI: 10.1063/1.2802038]
\end{abstract}

Magnetic data storage density is advancing with amazing speed, supported by the progress in both nanotechnology and nanomagnetism. ${ }^{1}$ The industry is presently aiming at densities toward and beyond $1 \mathrm{Tbit} / \mathrm{in}^{2}{ }^{2}$. Several challenges need to be addressed to realize this goal, one of which is the well known superparamagnetic limit of the recording media. ${ }^{3}$ As the bit and, therefore, the crystallite size of the thin film recording media shrinks to accommodate higher recording density, the thermal energy will eventually dominate the anisotropy energy, leading to grains with randomly fluctuating magnetization that are incapable of storing information.

Bit-patterned media has been proposed more than a decade ago, where each discrete identical nanodot is used to store a single bit. The superparamagnetic limit is applicable to a single bit rather than individual grains within a multigrain bit, thus leaving room for the further improvement of areal density. Cui et al. have used e-beam lithography to pattern magnetic thin films with areal density of 65 Gbits/in. ${ }^{2}{ }^{4}$ However, the top-down approach is slow and cost prohibitive for mass production. The development of the so-called self-organized magnetic array using solution phase synthesis and self-assembly of FePt nanoparticles have generated tremendous interest in recent years due to their low fabrication cost and the highest achievable density. ${ }^{5}$ However, prevention of particle aggregation during annealing ${ }^{6}$ and aligning of magnetic easy axis remain the two greatest hurdles for applications. An alternative bottom-up approach is to use self-assembled porous templates for the growth of magnetic arrays. ${ }^{7,8}$ Magnetic nanowire arrays have been produced by electrochemical deposition in porous anodic alumina (PAA) templates. ${ }^{8}$ However, high aspect ratio of the nanowires is not desirable for high density recording due to structural inhomogeneities, incoherent magnetization reversal, and limited writability. A recent paper reported $L 1_{0}$-ordered CoPt nanocolumns with $100 \mathrm{~nm}$ spacing and (001) orientation obtained by electrochemical deposition in

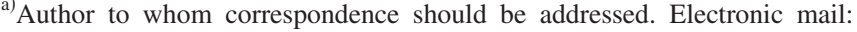
haozeng@buffalo.edu
}

PAA with postdeposition annealing. ${ }^{9}$ Magnetic and semiconductor nanodot arrays can also be fabricated by physical vapor deposition using ultrathin PAA templates as masks. ${ }^{10,11}$

Despite the rapid progress in this area, producing ordered magnetic nanodot arrays with very high density (spacing $<30 \mathrm{~nm}$ ), controlled easy axis orientation and high coercivity, which are essential for future storage media applications, remained a great challenge. In this paper, we report the development of $L 1_{0}$-structured FePt nanodot arrays with perpendicular anisotropy, high coercivity, and extremely high density. They were fabricated by physical vapor deposition using ultrathin PAA (Ref. 11) as masks, followed by rapid thermal annealing (RTA). There are several advantages of our systems comparing to early available materials: (1) the periodicity of $25 \mathrm{~nm}$ leading to extremely high density of above $1 \times 10^{12}$ dots/in. ${ }^{2}$, (2) large coercivity originating from the high magnetocrystalline anisotropy $\left(K_{u} \sim 2\right.$ $\times 10^{7} \mathrm{erg} / \mathrm{cm}^{3}$ ) of $L 1_{0}$-structured $\mathrm{FePt}$, (3) perpendicular easy axis orientation resulting from the (001) texture, ${ }^{12}$ (4) ease of fabrication without epitaxy. Thus, these nanodot arrays possess some of the most essential features desirable for future high density recording media.

PAA templates have been prepared by dc anodization of an aluminum foil in acidic solutions. ${ }^{13}$ Under appropriate conditions (acid type, concentration, temperature, and dc voltage), hexagonally close packed pore arrays with tunable diameter and periodicity can be formed. Figure 1 shows typical scanning electron microscope (SEM) images of PAA templates with different pore sizes and periodicities. Uniformity of the pore size and spacing is clearly demonstrated, as well as the spatial ordering. The standard deviation of the pore diameter is $\delta d / d<10 \%$. The highly ordered hexagonal pattern can extend up to a few micrometers. The domain size of an ordered region for the template with the smallest periodicity of $25 \mathrm{~nm}$ [Fig. 1(d)] is somewhat smaller. This can be improved by prepatterning the aluminum surface, e.g., with self-assembled monodisperse nanoparticles. ${ }^{14}$

To facilitate the penetration of atoms through the pores of the templates for the deposition of nanodots, ultrathin 


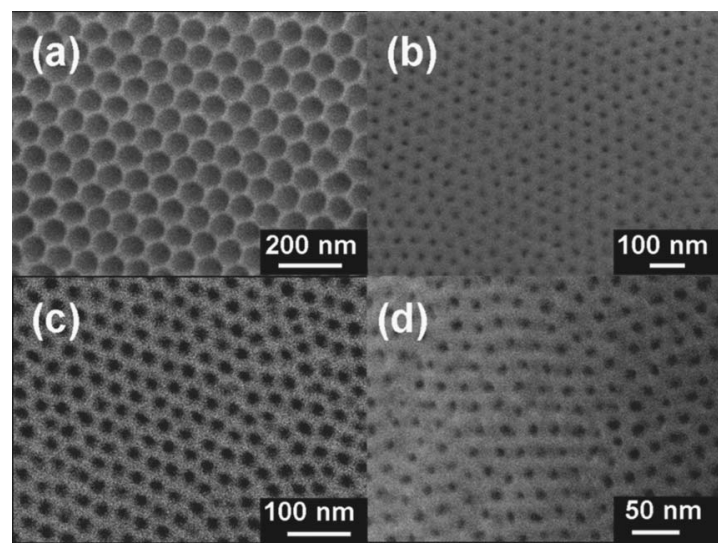

FIG. 1. Typical SEM images of porous alumina templates with different cell sizes and pore diameters: (a) 100 and $80 \mathrm{~nm}$, (b) 50 and $20 \mathrm{~nm}$, (c) 35 and $15 \mathrm{~nm}$, and (d) 25 and $12 \mathrm{~nm}$.

PAA templates have to be employed. This is realized by a two step anodization process: ${ }^{13}$ the first anodization between 2 and $12 \mathrm{~h}$ leads to pore arrays with long range order. The alumina layer was then removed by etching in acids to leave a pattern replicating the bottom of the pores on the aluminum surface, which acts as the pore nucleation sites for the second anodization. The second anodization time duration is tuned from 60 to $600 \mathrm{~s}$ to control the thickness of the templates between $500 \mathrm{~nm}$ and few micrometers. After the anodization, the top surface of the PAA templates was spincoated with a layer of polymethylmetacrylate (PMMA), and the aluminum and a thin nonporous barrier layer removed by immersing the templates in chemical etching solutions. ${ }^{11}$ Finally the templates were attached to glass or $\mathrm{Si} / \mathrm{SiO}_{2}$ substrates, dissolved of the PMMA coating, and cleaned and dried for the evaporation. Prior to removal of PMMA, the templates can be further thinned by immersing in phosphoric acid for different durations to bring the thickness of the templates down to $100 \mathrm{~nm}$, during which time the size of the pores are also somewhat enlarged. Figure 2 shows the typical SEM images of tilted views of the porous templates with different thicknesses, where the cross section reveals the thickness of the templates.

FePt nanodot arrays were prepared by sputtering $(\mathrm{Fe} / \mathrm{Pt})_{n}$ multilayers and removal of the PAA templates. The thickness of the Fe layer was varied while keeping the $\mathrm{Pt}$ layer-thickness constant, so that the composition could be adjusted. The nominal total thickness was varied from 50 to $200 \AA$ by changing the number of repetitions $n$. To realize the phase transformation of FePt, RTA was performed with temperatures between 500 and $700{ }^{\circ} \mathrm{C}$ with duration from 1 to 600 s. $^{12}$

The nanodot arrays faithfully replicate the porous structure of the templates. Figure 3(a) shows the typical SEM image of a highly ordered FePt nanodot array with period of $100 \mathrm{~nm}$ and diameter of $70 \mathrm{~nm}$, after RTA at $600{ }^{\circ} \mathrm{C}$ for $30 \mathrm{~s}$. It can be seen that the nanodot array has the same periodicity as that of the pore array and a diameter very similar to that of the template prior to deposition. The annealing does not change the size as well as the ordering of the nanodot array. While the dot and periodicity can be tuned by different electrolyte and anodization conditions, the dot size can be further adjusted without changing the periodicity by immersing the templates in weak acids to enlarge the pores. The top left corner in Fig. 3(a) is a partially removed

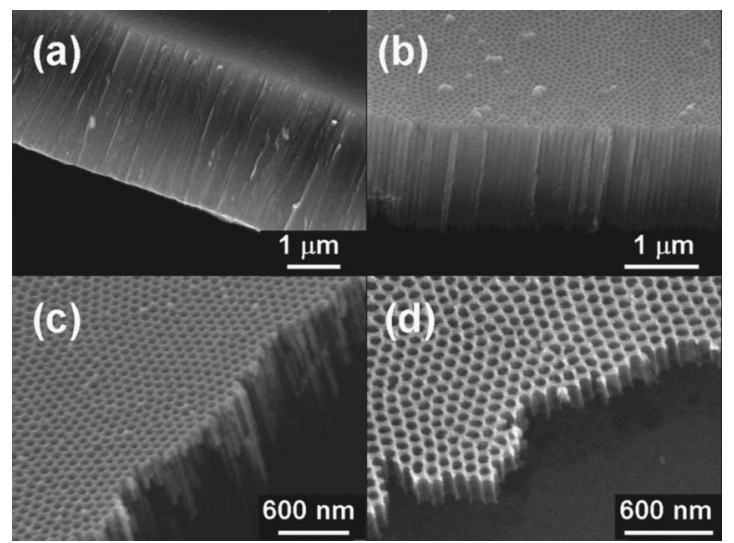

FIG. 2. SEM images of tilted views of porous alumina templates with thicknesses of (a) $2 \mu \mathrm{m}$, (b) $1 \mu \mathrm{m}$, (c) $600 \mathrm{~nm}$, and (d) $150 \mathrm{~nm}$.

template. It can be seen that after the deposition, the pores are partially covered by the deposited materials and appear smaller. Figure 3(b) shows the SEM image of an FePt nanodot array with diameter of $18 \mathrm{~nm}$ and period of $25 \mathrm{~nm}$, representing a density of $1.2 \times 10^{12} \mathrm{dots} / \mathrm{in}^{2}$. The nanodots are typically not of cylindrical or disklike shape, but rather resemble that of spherical caps. This is due to the closure effect and shadowing effect discussed in Ref. 11.

$L 1_{0}$-structured FePt nanodot arrays have been realized by RTA of the as-deposited Fe/Pt multilayer nanodot arrays. $L 1_{0}$-structured FePt has a face centered tetragonal structure with the $\mathrm{Fe}$ and $\mathrm{Pt}$ atoms stacking alternately along the $c$ axis. For fully $L 1_{0}$-ordered FePt, the low crystal symmetry and strong spin-orbit coupling can lead to very high $K_{u}$ of the order of $5 \times 10^{7}-1 \times 10^{8} \mathrm{erg} / \mathrm{cm}^{3}$ with the easy axis oriented along the (001) axis. ${ }^{15}(001)$-textured FePt thin films give perpendicular anisotropy and coercivity, which are normally achieved by epitaxial growth on single crystal substrates. ${ }^{16}$ Following our previous work on thin films, ${ }^{12}$ we have realized (001)-textured FePt nanodot arrays without epitaxy using RTA. Figure 3(c) shows the $\mathrm{x}$-ray diffraction (XRD) pat-
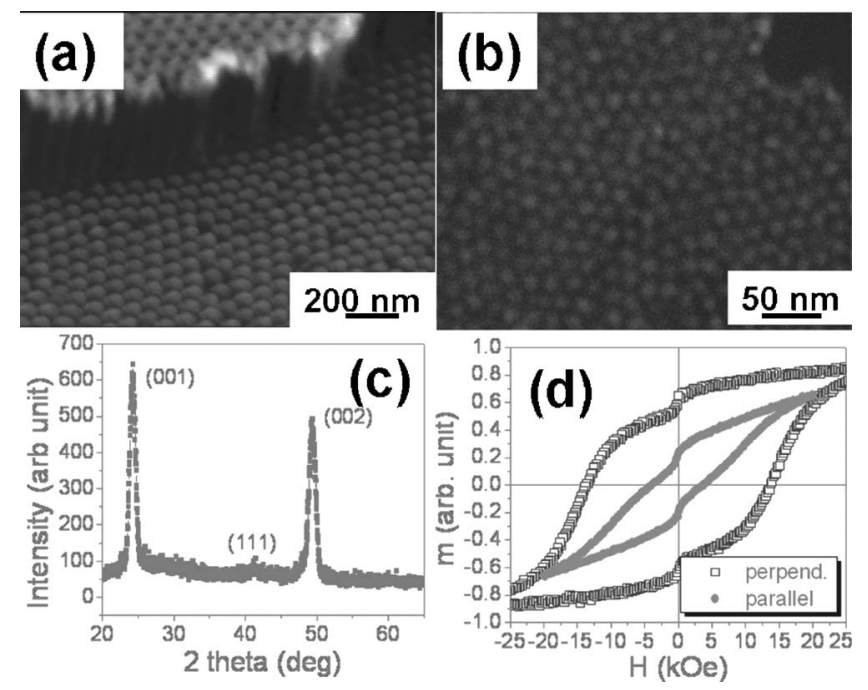

FIG. 3. SEM images of (a) An FePt nanodot array with cell size of $100 \mathrm{~nm}$, diameter of $70 \mathrm{~nm}$ and nominal thickness of $100 \AA$ (top-left corner is the partially remaining porous template); (b) an FePt nanodot array with diameter of $18 \mathrm{~nm}$ and periodicity of $25 \mathrm{~nm}$; (c) the XRD pattern of an FePt nanodot array with $100 \mathrm{~nm}$ periodicity and $70 \mathrm{~nm}$ dot size, after RTA at $600{ }^{\circ} \mathrm{C}$ for $30 \mathrm{~s}$; (d) the $300 \mathrm{~K}$ magnetic hysteresis loops of the nanodot array measured at both parallel and perpendicular to the substrate directions. 
tern of a $70 \mathrm{~nm}$ FePt nanodot array on glass substrate annealed at $600{ }^{\circ} \mathrm{C}$ for $30 \mathrm{~s}$. The sample is highly (001) oriented, as can be seen from the strong (001) and (002) superlattice peaks. Nearly all other peaks are absent except a weak bump corresponding to the (111) peak. As reported in our previous paper, ${ }^{12}$ the texture evolution process likely takes place in two distinctive stages, with different time scales. The first stage, initial nucleation of disordered fcc (100) nanocrystallites, occurs in a few seconds. This is presumably due to the interaction between the FePt and the glass substrate, e.g., by in-plane stress/strain. ${ }^{17}$ The second stage, the disorder-order transition, takes $30 \mathrm{~s}$ to a few minutes involving neighboring $\mathrm{Fe}$ and $\mathrm{Pt}$ atoms rearranging their respective positions. The disordered FePt with (100) orientation could in principle result in both (100) and (001) orientations in the $L 1_{0}$ phase. However, since the $c$ axis is slightly shorter than the $a$ axis, (001) orientation is preferred to lower the total surface energy. Thicker films have a tendency toward the closest packed (111) orientation due to weaker influence from the substrate. Prolonged annealing also favors grain growth and the development of (111) texture. Therefore, film thickness and annealing time are two critical factors in achieving highly oriented (001) texture.

The nearly perfect (001) texture should lead to aligned magnetic easy axis perpendicular to the plane. Figure 3(d) shows the magnetic hysteresis loops measured at $7 \mathrm{~T}$, in directions both parallel and perpendicular to the substrate. It is clearly seen that the easy axis lies in the perpendicular direction, which is consistent with XRD results. By extrapolating and intersecting the perpendicular and parallel hysteresis loops, we estimate a $K_{u}$ value of $2 \times 10^{7} \mathrm{erg} / \mathrm{cm}^{3}$. This is lower than that of fully ordered FePt due to the short annealing time and partial ordering. The perpendicular loop shows large coercivity of $15 \mathrm{kOe}$, owing to the large perpendicular anisotropy of the material. The hard axis loop still exhibits substantial coercivity, which is due to a slight misalignment of the easy axis. A small kink appears at the zero field, which is possibly related to off-stoichiometry and/or incomplete phase transformation due to short annealing time. ${ }^{18}$

While the present systems address several major challenges in self-assembled magnetic arrays, more research is needed to make them really feasible for patterned media applications. Heat-assisted recording may be used to overcome the write field limit of the head material. Long-range ordering and the symmetry of the nanodots are another issue. It has been demonstrated very recently that PAA templates with long-range order and sub-30 nm periodicity can be obtained, e.g., by prepatterning the aluminum with a two-dimensional array of self-assembled nanoparticles. ${ }^{14,19}$ It is not yet clear whether one may be able to induce circumferentially ordered nanodots by prepatterning to preserve the current scheme of flying a head above the media. The uniformity in magnetic properties of individual dots also needs to be characterized and used for the guide to improve fabrication. It is of interest to give a realistic estimate of the ultimate areal density of the patterned media with the current approach: assuming the bulk $K_{u}$ value of $5 \times 10^{7} \mathrm{erg} / \mathrm{cm}^{3}$ and thermal stability parameter $\alpha=K_{u} V / k T=50$, a simple estimate of the superparamagnetic size limit for a spherical (the aspect ratio of nanodots cannot be much higher than 1 due to the fabrication limit) FePt nanoparticle is about $4.4 \mathrm{~nm}$. Considering a possible surface spin disorder and loss of magnetic moment at the particle surface, a more conservative size limit should be about $5 \mathrm{~nm}$. Assuming a periodicity of $6 \mathrm{~nm}$, one obtains an ultimate density of $20 \mathrm{Tbits} / \mathrm{in}^{2}$. Densities beyond this must rely on a paradigm drastically different from the current ones.

In summary, we have demonstrated the fabrication of ordered FePt nanodot arrays with tunable dimensions by physical vapor deposition through PAA templates. The periodicity as small as $25 \mathrm{~nm}$ has been achieved, leading to an areal density of $1.2 \times 10^{12}$ dots/in. ${ }^{2}$. The easy axis orientation can be controlled to achieve perpendicular anisotropy and very large coercivity. These systems possess several essential features desirable for future magnetic data storage media applications. Magnetic nanodot arrays including Co, $\mathrm{Ni}$, and CoPt with various dimensions have also been fabricated with the same technique. These arrays are ideal model systems to test theories of nanomagnetism. The technique can be extended to the fabrication of electronic and optical nanodevices with extremely small size and high density.

This work is supported at Buffalo by NSF DMR 0547036 and UB MIPS grant, and at Nebraska by NSFMRSEC (DMR-0213808), INSIC and the Nebraska Center for Materials and Nanoscience.

${ }^{1}$ S. D. Bader, Rev. Mod. Phys. 78, 1 (2006).

${ }^{2}$ H. J. Richter, A. Y. Dobin, O. Heinonen, K. Z. Gao, R. J. M. Van der Veerdonk, R. T. Lynch, J. Xue, D. Weller, P. Asselin, M. F. Erden, and R. M. Brockie, IEEE Trans. Magn. 42, 2255 (2006).

${ }^{3}$ D. Weller and A. Moser, IEEE Trans. Magn. 35, 4423 (1999).

${ }^{4}$ B. Cui, W. Wu, L. S. Kong, X. Y. Sun, and S. Y. Chou, J. Appl. Phys. 85, 5534 (1999).

${ }^{5}$ S. H. Sun, C. B. Murray, D. Weller, L. Folks, and A. Moser, Science 287, 1989 (2000).

${ }^{6}$ H. Zeng, S. H. Sun, T. S. Vedantam, J. P. Liu, Z. R. Dai, and Z. L. Wang, Appl. Phys. Lett. 80, 2583 (2002).

${ }^{7}$ T. Thurn-Albrecht, J. Schotter, C. A. Kastle, N. Emley, T. Shibauchi, L. Krusin-Elbaum, K. Guarini, C. T. Black, M. T. Tuominen, and T. P. Russell, Science 290, 2126 (2000).

${ }^{8}$ H. Zeng, M. Zheng, R. Skomski, D. J. Sellmyer, Y. Liu, L. Menon, and S. Bandyopadhyay, J. Appl. Phys. 87, 4718 (2000); H. Zeng, R. Skomski, L. Menon, Y. Liu, D. J. Sellmyer, and S. Bandyopadhyay, Phys. Rev. B 65, 134426 (2002)

${ }^{9}$ N. Yasui, A. Imada, and T. Den, Appl. Phys. Lett. 83, 3347 (2003).

${ }^{10}$ K. Liu, J. Nogues, C. Leighton, H. Masuda, K. Nishio, I. V. Roshchin, and I. K. Schuller, Appl. Phys. Lett. 81, 4434 (2002).

${ }^{11}$ Y. Lei and W. K. Chim, Chem. Mater. 17, 580 (2005).

${ }^{12}$ H. Zeng, M. L. Yan, N. Powers, and D. J. Sellmyer, Appl. Phys. Lett. 80, 2350 (2002).

${ }^{13}$ H. Masuda, H. Yamada, M. Satoh, H. Asoh, M. Nakao, and T. Tamamura, Appl. Phys. Lett. 71, 2770 (1997).

${ }^{14}$ Y. Matsui, K. Nishio, and H. Masuda, Small 2, 522 (2006).

${ }^{15}$ D. Weller, A. Moser, L. Folks, M. E. Best, W. Lee, M. F. Toney, M. Schwickert, J. U. Thiele, and M. F. Doerner, IEEE Trans. Magn. 36, 10 (2000).

${ }^{16}$ R. F. C. Farrow, D. Weller, R. F. Marks, M. F. Toney, A. Cebollada, and G. R. Harp, J. Appl. Phys. 79, 5967 (1996).

${ }^{17}$ J. S. Kim, Y. M. Koo, B. J. Lee, and S. R. Lee, J. Appl. Phys. 99, 053906 (2006).

${ }^{18}$ J. P. Liu, C. P. Luo, Y. Liu, and D. J. Sellmyer, Appl. Phys. Lett. 72, 483 (1998).

${ }^{19}$ H. Masuda, K. Takenaka, T. Ishii, and K. Nishio, Jpn. J. Appl. Phys., Part 2 45, L1165 (2006). 